\title{
Empirical Ultra Wide Band Channel Model for Short Range Outdoor Environments
}

\author{
Jinwon Choi, Noh-Gyoung Kang, Yu-Suk Sung and Seong-Cheol Kim \\ School of Electrical Engineering and INMC, Radio Technology Lab., \\ Seoul National University, Seoul, Korea \\ E-Mail : caesar@maxwell.snu.ac.kr, sckim@maxwell.snu.ac.kr
}

\begin{abstract}
This paper reports the empirical Ultra Wide Band (UWB) channel model for short range outdoor environments. The measurements were performed based on the frequency sweep method in frequency domain at two different parking lot environments. From the measured channel transfer functions, parameters of log-distance path loss law and the effect of frequency to the path loss exponent are extracted. The distribution of received signal is analyzed to the existence of line-of-sight (LOS) path also. For the characterization of frequency dependent UWB channel parameters, standard deviation and correlation properties of channel gain in frequency domain are analyzed. The standard deviation increases with transmitter-receiver separation in LOS condition and the correlation is regressed to the double-slope model.
\end{abstract}

Keywords - Ultra WideBand (UWB), Empirical Channel Model, Outdoor UWB and Channel Measurement.

\section{INTRODUCTION}

The Ultra Wide Band (UWB) system has drawn one's interest as a new short range high data rate wireless indoor communication system. The advantages of UWB, such as low complexity, low power, resistance to multi-path and very good time domain resolution, are essential motivations in spite of disadvantages, i.e. RF design and interference problem. To accentuate the advantages and diminish the disadvantages, the exact channel model for UWB system is necessary. Many researches about UWB radio channel have been in progress and literature reported about the distinguishable UWB channel characteristics for efficient systems engineering and interference problem [2] [10]. However, few work deal with about the outdoor UWB channel, because the UWB is considered as the system for the indoor environments only [1]. To design the UWB systems engineering of short range outdoor environments and to predict the interference due to the radiation from the systems deployed indoor to the outdoor communications, the channel characterization work for UWB system of outdoor environments has to be analyzed.

In this paper, we investigated the empirical UWB channel model in frequency domain at short range parking lot environments. The parking lot environment is selected for channel characterization measurement because the car could be related to the wireless communication services like location based services (LBS). The measurements are performed using the frequency sweep method with the vector network analyzer (VNA) [9,11]. From the measured channel transfer functions
(CTFs), the path loss behavior of UWB in outdoor environments is reported at first. The path loss characteristics are the most fundamental parameter among the radio propagation characteristics to develop the wireless communication systems and predict the interference to other communications. For UWB system, it is more emphasized because the UWB is expected to co-exist with many other systems and its large frequency bandwidth is the underlying suspect for interference problem. So, the practical path-loss parameters of log-distance path loss law for outdoor UWB systems are studied. Furthermore, the effect of frequency to path-loss behavior and the distributions of received UWB signal with the existence of LOS path are accomplished. For the characterization of frequency dependent channel properties, the standard deviation and correlation of channel gain in frequency domain are investigated.

This paper is organized as follows. Section 2 presents the channel measurement system and measurement scenario. Section 3 provides the UWB path loss properties and section 4 does the statistical analysis about channel gain of frequency domain. Finally, the conclusion is followed in section 5.

\section{Measurement CAmpaign}

\section{A. Measurement system}

Among UWB channel measurement methods, we selected the frequency domain channel sounding method for channel characterization $[9,11]$. In this measurement technique, wide frequency bands are swept using a set of narrow-band signals and channel frequency responses are recorded using a vector network analyzer (VNA). A VNA (Agilent 8719ES) transmits 801 continuous wave tones distributed uniformly from $5 \mathrm{GHz}$ $6.6 \mathrm{GHz}$ with the frequency separation of $2 \mathrm{MHz}$. The $5 \mathrm{GHz}$ frequency band is selected for the channel characterization considering the coexistence with the 802.11 a wireless local area system. Though different wireless systems share the frequency band, the coexistence and interference problem can be solved through the accurate channel model. $2 \mathrm{MHz}$ frequency interval allows us to capture multi-paths with the maximum excess delay of 500 nano-seconds and the bandwidth of $1.6 \mathrm{GHz}$ gives the delay resolution of 0.625 nano-seconds. The path loss of up to $110 \mathrm{~dB}$ can be compensated to guarantee the proper input power to VNA by a power amplifier and a step attenuator. The transmitting and receiving antennas are omni-directional with $2.1 \mathrm{dBi}$ gain.

This work is supported in part by University IT Research Center project and Seoul R\&BD Program 


\section{B. Measurement Scenario}

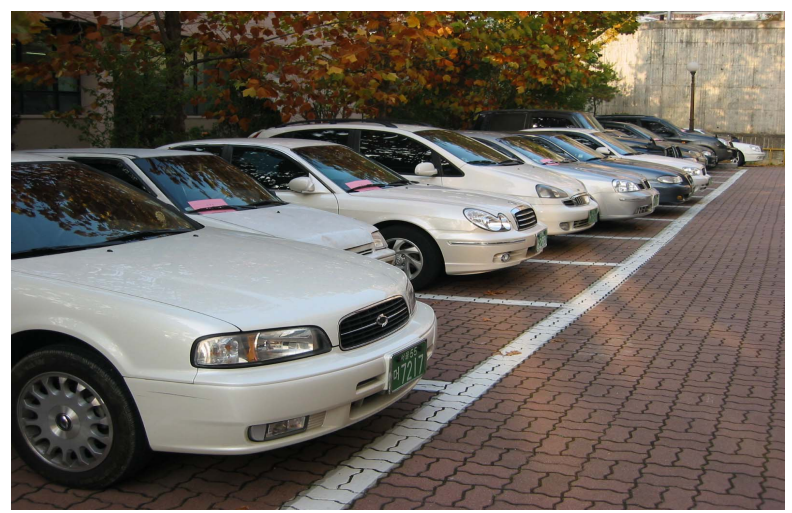

(a) Picture of Measurement Environment 1

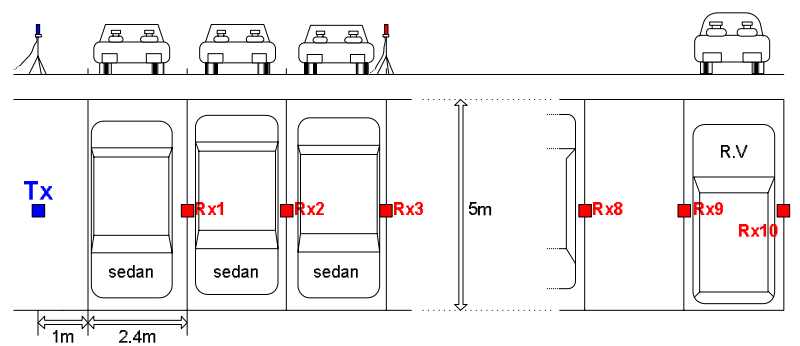

(b) Tx and Rx locations at Environment 1

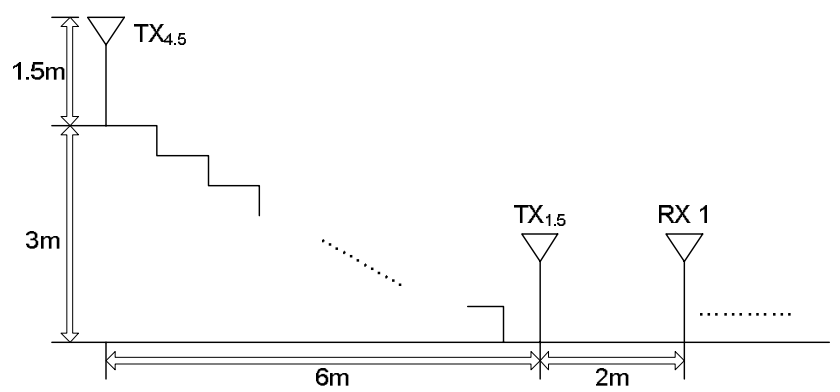

(c) Side view of Tx and Rx locations at Environment 2

Figure 1. Description of parking lot environments and $\mathrm{Tx}, \mathrm{Rx}$ locations where measurements performed

The frequency sweep measurements are carried out at two different parking lot environments in Seoul National University, Korea. The descriptions about measurement environments and locations of transmitting and receiving antennas are illustrated in figure 1 . The $1^{\text {st }}$ parking lot, which is called Environment 1, is located behind the building and surrounded by buildings and walls. In Environment 1, the receivers are distributed linearly to characterize the effect of parked cars. The measurements are performed when there is no parked car and when the cars are parked. The heights of both antennas are $1.5 \mathrm{~m}$.

The $2^{\text {nd }}$ parking lot, called Environment 2, is situated in front of the stairs of 15 stories building. In this environment, the transmitter (Tx) is located at different height, $1.5 \mathrm{~m}$ and $4.5 \mathrm{~m}$. There are 22 receiver $(\mathrm{Rx})$ positions in both transmitter heights of Environment 2. The furthest receiver is $31 \mathrm{~m}$ away from the
$4.5 \mathrm{~m}$ transmitter, denoted as $\mathrm{Tx}_{4.5}$, respectively $28 \mathrm{~m}$ for transmitter with $1.5 \mathrm{~m}$ height, $\mathrm{Tx}_{1.5}$. When the measurement is performed in Environment 2, there are no parked car, pedestrian and obstacle between all transmitter-receiver cases. In single receiver position, 200 channel transfer functions (CTFs) are collected and total 12,800 CTFs are analyzed for the UWB channel characterization.

\section{PATH LOSS PROPERTIES FROM MEASURED DATA}

\section{A. Empirical path loss parameters}

From the path loss properties, fundamental system parameters, such as link budget, cell planning and interference prevention technique, would be decided. For the characterization of UWB path loss behavior, the log-distance path loss law is used. The widely known formula is given in Equation (1) where $\operatorname{PL}\left(\mathrm{d}_{0}\right)$ means path loss at the reference distance $\mathrm{d}_{0}(=1 \mathrm{~m})$, $\mathrm{d}$ is separation between the transmitter and receiver, $\mathrm{n}$ is the path loss exponent and $\mathrm{S}$ is a Gaussian-distributed random variable with zero mean and standard deviation $\sigma_{\mathrm{s}}$.

$$
P L(d)=P L\left(d_{0}\right)+10 n \log \left(d / d_{0}\right)+S
$$

The measured results of 4 receiver environments are drawn with in figure 2. The numerical values of path loss exponents and the standard deviation of shadowing factor values given in Table 1 are obtained through the minimum mean square error (MMSE) algorithm. Using the power amp and step attenuator at transmitter stage, the radiation power of measurement system is set to satisfy the announced FCC UWB regulation.

Table 1. The empirical path loss parameters to receiver conditions

\begin{tabular}{|c||c|c|c|}
\hline & $\mathrm{PL}\left(\mathrm{d}_{0}\right)[\mathrm{dB}]$ & $\mathrm{n}$ & $\sigma_{\mathrm{s}}[\mathrm{dB}]$ \\
\hline \hline LOS (Environment1) & -47.985 & 1.78 & 1.113 \\
\hline $\mathrm{CAR}$ (Environment 1) & -47.618 & 3.02 & 3.592 \\
\hline $\mathrm{Tx}_{1.5}$ (Environment 2) & -43.425 & 2.21 & 0.714 \\
\hline $\mathrm{Tx}_{4.5}$ (Environment 2) & -43.425 & 2.19 & 2.400 \\
\hline
\end{tabular}

n: path loss exponent $\quad \sigma_{\mathrm{s}}$ :the standard deviation of shadowing factor

For environment 1, the frequency averaged path loss exponent is 1.78 in LOS locations and 3.02 when the cars are parked. At environment 2, the path loss exponent values are about 2.2 whether height of antennas is same or not. It is observed that the height difference between transmitting antenna and receiving antenna does not have effect on the path loss exponent value. However, in case of shadowing factor, the standard deviation of shadowing factor $\mathrm{S}$ gets bigger when the height of transmitting and receiving antenna is different. The $\sigma_{\mathrm{s}}$, standard deviation of shadowing factor $\mathrm{S}$ gets bigger from $0.714 \mathrm{~dB}$ to $2.4 \mathrm{~dB}$ as the transmitter gets higher in Environment 2 . 


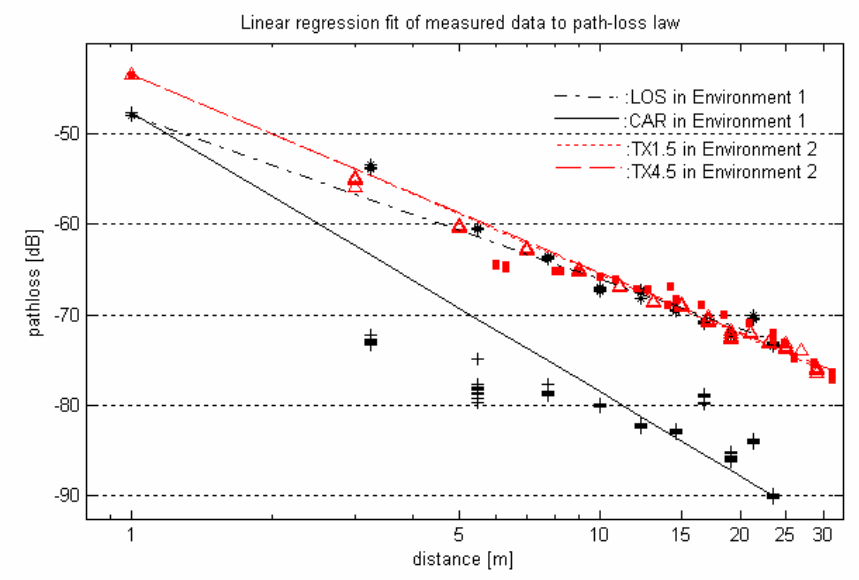

Figure 2. Linear regression fit of measured data to log distance path-loss law in each propagation environment

\section{B. Effect of Frequency on path loss properties of UWB signal}

The Multi-Band Orthogonal Frequency Division Multiplexing (MB-OFDM) scheme proposes that the assigned frequency range for UWB system operation would be divided into sub-bands with about $500 \mathrm{MHz}$ bandwidth [13]. For the intelligent MB-OFDM system, the different path loss parameters are applied as the allocated frequency band is changed.

To analyze the effect of frequency on path loss properties of UWB signal, frequency range of our measurement, $1.6 \mathrm{GHz}$ bandwidth at $5.8 \mathrm{GHz}$ band, is swept by sliding the frequency window of $500 \mathrm{MHz}$ bandwidth with the discrete interval of $100 \mathrm{MHz}$. The statistical values of path loss exponents averaged over $500 \mathrm{MHz}$ bandwidths are summarized in Table 3 . As in indoor environments [9], the path loss exponent increases with center frequency. The amount of variation is bigger in LOS case than in CAR case of environment 1 and in $\mathrm{Tx}_{4.5}$ case than in $\mathrm{Tx}_{1.5}$ case of environment 2 .

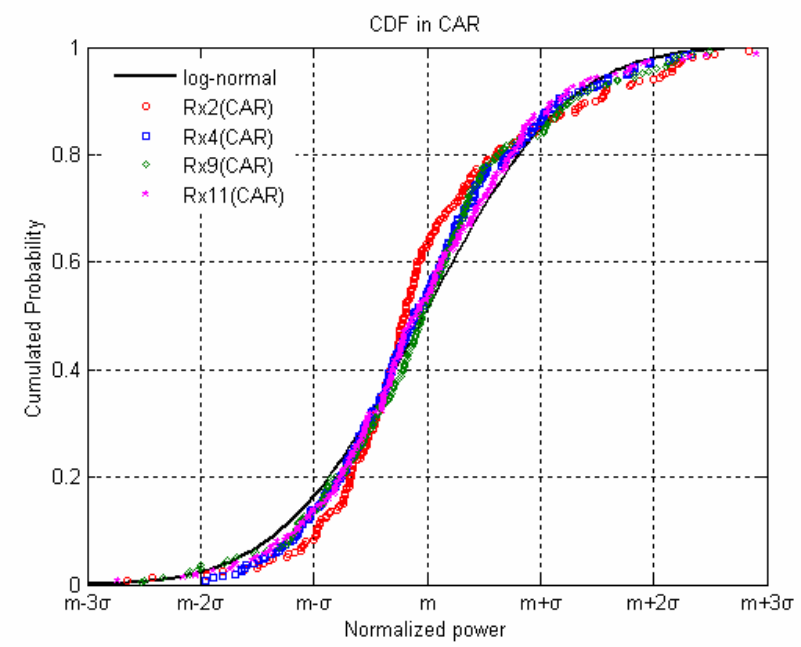

Table 2. Path loss exponent variation of 500MHz frequency bands

\begin{tabular}{|c||c|c|c|c|c|}
\hline & $\mathrm{n}_{1.6 \mathrm{GHz}}$ & $\operatorname{Min}\left(\mathrm{n}_{500}\right)$ & $\operatorname{Max}\left(\mathrm{n}_{500}\right)$ & $\Delta\left(\mathrm{n}_{500}\right)$ & $\Delta\left(\mathrm{n}_{500}\right) / \mathrm{n}_{1.6 \mathrm{GHz}}$ \\
\hline \hline LOS & 1.78 & 1.60 & 2.01 & 0.41 & 0.25 \\
\hline CAR & 3.02 & 2.96 & 3.23 & 0.27 & 0.09 \\
\hline $\mathrm{Tx}_{1.5}$ & 2.21 & 2.17 & 2.29 & 0.12 & 0.05 \\
\hline $\mathrm{Tx}_{4.5}$ & 2.19 & 2.04 & 2.32 & 0.28 & 0.13 \\
\hline \hline Outdoor & 2.27 & 2.16 & 2.42 & 0.26 & 0.11 \\
\hline Indoor [9] & 2.06 & 1.74 & 2.44 & 0.70 & 0.34 \\
\hline
\end{tabular}

In comparison with the results of indoor environments, the margin of path loss exponent is smaller than that of indoor environments though the path loss exponent over $1.6 \mathrm{GHz}$ of outdoor environments is bigger. This is because the outdoor environment is much simpler than indoor environment, especially various structure materials

\section{Distribution of UWB received signal}

To understand the path loss properties of UWB signal properly, not only the average path loss characteristics but also the distribution of the received signal power has to be analyzed. Figure 3 illustrates the cumulative distribution functions (CDFs) of received signal of CAR and LOS locations in Environment 1. In Figure 3, x-axis indicates the normalized power to averaged received power and y-axis means the cumulated probability. In CAR case, CDFs of received UWB signal are similar to the Gaussian distribution when the measured signal have values in $\mathrm{dB}$ unit. This means that the received signal of CAR case follows the log-normal fading [12]. In LOS case, the CDF is similar to the Nakagami-m fading with $\mathrm{m}$ is 2.4 .

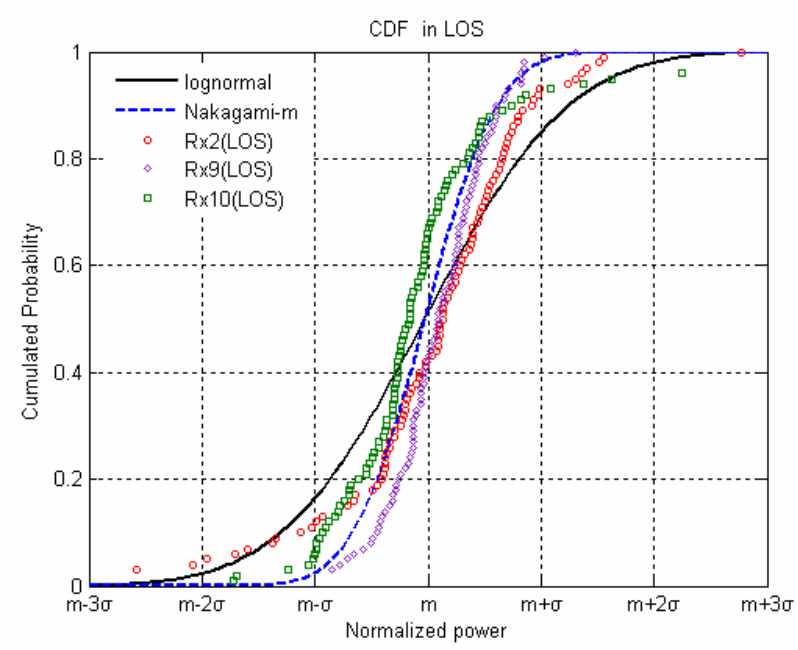

Figure 3. Received signal CDF in CAR and LOS cases at Environment 1 


\section{FREQUENCY DEPENDENT CHANNEL PARAMETERS}

\section{A. STDEV of channel gain with frequency}

Using the path loss properties, the average signal strength at a receiver and the distributions of UWB signal are predictable for the systems engineering. Add to these parameters, the variation of the propagated UWB signal with frequency is useful for receiver design in frequency domain. For the analysis on the frequency dependent channel characteristics, we expressed the frequency response of the UWB radio channel $h(f)$ as

$$
h(f)=\sum_{f_{k}=f_{\min }}^{f_{\max }} a\left(f_{k}\right) e^{j \theta\left(f_{k}\right)}\left(f-f_{k}\right)
$$

where $a\left(f_{k}\right)$ is the channel amplitude gain of frequency $f_{k}$ and $\theta\left(f_{k}\right)$ is the phase respectively. Based on this representation, the standard deviation of received frequency tones (STDEV) is defined as (3).

$$
\mathrm{STDEV}=\sigma\left\{a\left(f_{k}\right)\right\}[\mathrm{dB}]
$$

The term STDEV indicates the received signal power spread of frequency tones in $\mathrm{dB}$ scale. From the STDEV, the UWB receivers predict the dispersiveness of UWB signal to frequency. In Table 3, the representative values of STDEV are expressed numerically and increase of STDEV to the Tx-Rx separation in LOS locations when the height of transmitting antenna is same to that of receiving one is illustrated in figure 4 . When the LOS path is guaranteed, the STDEV increase with slope $0.1 \sim 0.15$ as separation grows and the average of STDEV in LOS locations is $3.75 \mathrm{~dB}$. For NLOS locations, the average of STDEV is $5.98 \mathrm{~dB}$, which is almost same to the maximum value among the STDEVs of LOS locations. Furthermore, STDEV of NLOS locations does not vary much, as standard deviation of STDEV is only $0.23 \mathrm{~dB}$ while $1.16 \mathrm{~dB}$ in LOS locations. This means that the channel gain to frequency gets dispersive once the LOS path is interrupted and it does not depend on the separation in NLOS case.

Table 3. Numerical expression of STDEV

\begin{tabular}{|c||c|c|c|c|}
\hline$[\mathrm{dB}]$ & Mean & Std. & Max. & Min. \\
\hline \hline LOS & 3.75 & 1.16 & 5.97 & 1.60 \\
\hline NLOS & 5.98 & 0.23 & 6.23 & 5.56 \\
\hline
\end{tabular}

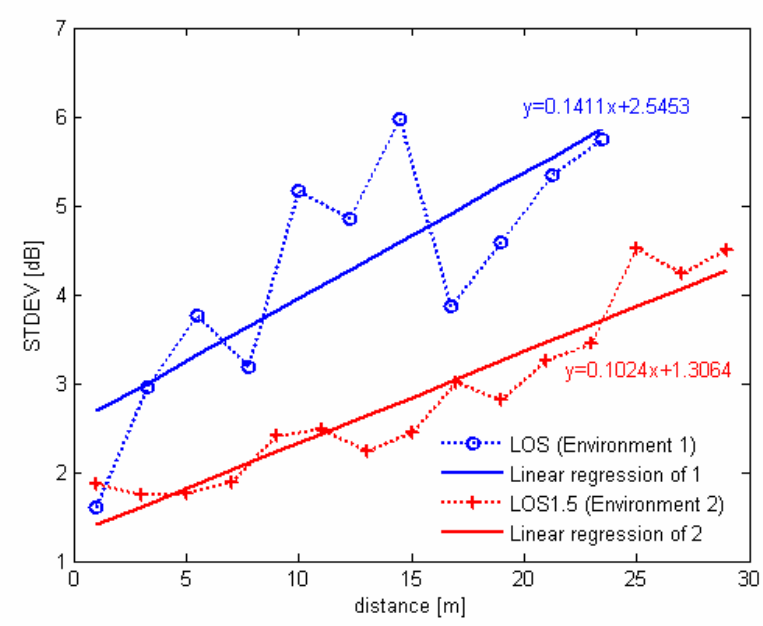

Figure 4. STDEV to the Tx-Rx separation in LOSs

\section{B. Correlation of received UWB signal}

The frequency dependent UWB channel variation and the interference level to out-of-band can be predictable from the correlation property. In this paper, the correlation coefficient is used for representing the correlation of channel gain of each frequency tone. The correlation coefficients are calculated using the formula in [14] and represented in Eq. 4.

$$
\rho_{a}(\Delta f)=\frac{C_{a}(f, f+\Delta f)}{\sqrt{C_{a}(f, f)} \sqrt{C_{a}(f+\Delta f, f+\Delta f)}}
$$

where

$$
C_{a}\left(f_{1}, f_{2}\right)=E\left[\left\{a\left(f_{1}\right)-m_{a}\left(f_{1}\right)\right\}\left\{a\left(f_{2}\right)-m_{a}\left(f_{2}\right)\right\}\right]
$$

$a\left(f_{1}\right)$ : channel amplitude gain of frequency tone $f_{1}$

$m_{a}\left(f_{1}\right)$ : mean of $a\left(f_{1}\right)$

Figure 3 illustrates the correlation coefficient values to the frequency separation whether cars are parked or not in Environment 1. The correlation coefficient to frequency separation is regressed to double-slope model. The break point of double-slope model is $50 \mathrm{MHz}$ and coefficients of double-slope model are represented in Table 4. In location with LOS path between same-height antennas, the coefficients decline rapidly rather than other locations. As the frequency separation goes above $50 \mathrm{MHz}$, the coefficients are converged to under 0.1 in all locations. 


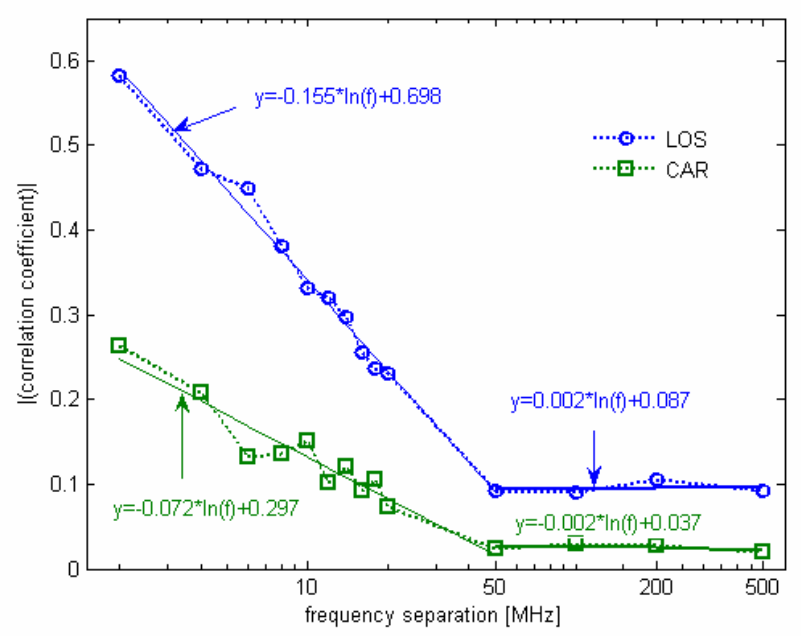

Figure 5. Correlation coefficients to the frequency separation in Environment 1 (dashed line: calculated value, solid line : double-slope model)

Table 4. Parameters of correlation coefficient to frequency separation double-slope model

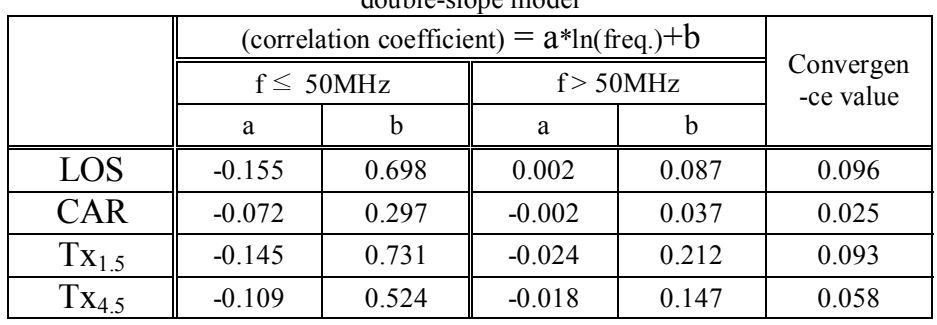

\section{Conclusion}

For UWB system performance, the experimental UWB channel characterization, which is crucial to estimate, is carried out in short range outdoor parking lot environments. The measurement system used for channel characterization adopts the frequency sweep method. The path loss exponent of log distance path loss formula is around 2 when the LOS path is guaranteed and just over 3 for NLOS locations. In study of the frequency dependency to the UWB signals, amount of path loss exponent variation to frequency is smaller than that of indoor environments. The CDF of received UWB signal follows log-normal distribution in NLOS case and Nakagami-m fading in LOS case. The channel gain of frequency domain gets more dispersive as the separation grows when LOS path is guaranteed. Finally, the correlation coefficient between channel gains is modeled to the double-slope model.

\section{REFERENCES}

[1] Win, M.Z. Ramirez-Mireles, F, Scholtz, R.A and Barnes, M.A "A path link model for ultra wide band pulse transmissions" VTC 1997, vol. 1, pp.251-255, 4-7 May 1997.

[2] Cassioli, D., Win, M.Z. and Molisch, A.F., "The ultra-wide bandwidth indoor channel: from statistical model to simulations," IEEE Journal on Selected Areas in Communications, vol. 20, Issue 6, pp.1247-1257, Aug. 2002

[3] K. Siwiak, "A path link model for ultra wide band pulse transmissions" VTC 2001 Spring, vol. 2, pp.1173-1175, May 2001.

[4] S.S Ghassemzadeh, L.J. Greenstein, A. Kavcic, T. Sveinsson and V. Tarokh, "UWB Indoor Path Loss Model for Residental and Commercial buildings," in Proc. IEEE Veh. Technol. Conf. (VTC 200-Fall), vol. 5, pp.3115-3119, Oct 2003.

[5] R.A. Scholtz et al, "UWB radio deployment challenges." PIMRC 2000, vol. 1, pp.620-625, Sep. 2000

[6] Uguen, B., Plouhinec, E., Lostanlen, Y., Chassay, G., "A deterministic ultra wideband channel modeling," IEEE Conference on Ultra Wideband Systems and Technologies, pp. 1-5, May 2002.

[7] Ramirez-Mireles, F., "On the capacity of UWB over multipath channels," IEEE Communications Letters, vol 9, Issue 6, pp. 523-525, Jun 2005

[8] Chong, C.-C., Yong, S.K., "A Generic Statistical-Based UWB Channel Model for High-Rise Apartments," IEEE Transactions on Antennas and Propagation, vol. 53, Issue 8, Part 1, pp. 2389-2399, Aug. 2005

[9] Jinwon Choi, Yu-Suk Sung, Noh-Gyoung Kang and S.C.Kim, "Empirical Ultra Wide Band Path loss model in office environments," VTC 2006 Spring, vol. 4, pp.1956-1960, May 2006.

[10] Irahhauten, Z., Nikookar, H., Janssen, G.J.M., "An overview of ultra wide band indoor channel measurements and modeling," IEEE Microwave and Wireless Components Letters, vol. 14, Issue 8, pp.386-388, Aug. 2004

[11] I. Oppermann. et al, UWB Theory and Applications, Wiley, England, 2004.

[12] Theodore S. Rappaport, Wireless communications: principles and practice $2^{\text {nd }}$ edition, Prentice Hall PTR, Upper Saddle River, NJ, USA, 2002

[13] http://www.multibandofdm.org

[14] Leon Garcia, Probability and Random Processes for Electrical Engineering $2^{\text {nd }}$ edition, Addison Wesley, NY, USA, 1994 